\title{
Evaluation of Topography Retrieval of Exo Mars Using Single Image DTM Estimation and Super Resolution Restoration Method-Review
}

\author{
P.Suresh Kumar ${ }^{1}$, G.Nirmala ${ }^{2}$, S.Manimegalai ${ }^{3}$, H.Arulvedi ${ }^{*}$ \\ 1 Department of EEE, Mahendra Engineering College (Autonomous), Mahendhirapuri, \\ Mallasamudram - 637 503, Tamil Nadu, India. \\ 2Department of ECE,Mahendra Institute of Technology, Mahendhirapuri, \\ Mallasamudram - 637 503,Tamil Nadu, India. \\ ${ }^{3}$ Department of ECE, Mahendra Engineering College for Women, \\ Tiruchengode, Namakkal 637205, India
}

\begin{abstract}
This review examines the meta-data of high-resolution orbital imagery obtained by Mars during the last four decades. The aim of this study is to provide a starting point for planetary scientists interested in exploring the martian surface for modifications linked to natural phenomena. A framework for generating picture groupings relevant to prioritising regions for shift detection is adopted and used for analysis. The season, the Martian Year, and the local period that an image was captured, as well as the imaging device and its resolution, are the criteria that determine each grouping. The present work indicates that there is enough coverage to regularly analyze periodic martian phenomena in images depicting the same region during the same season, as well as intermittent martian phenomena in images depicting the same area in various time periods. While with this treatment of the human visual system is short, it offers a simple understanding of the eye's capabilities in perceiving pictorial details. To clean up the noise and other defects in the data, to improve subtle information not noticeable in batch-processed NASA photos were discussed. To magnify smaller features for study, the used fairly standard image processing techniques.
\end{abstract}

KEYWORDS: Mars, Space, Mapping,Image Processing Techniques

https://doi.org/10.29294/IJASE.8.3.2022.2323-2327

(C2022 Mahendrapublications.com, All rights reserved

\section{INTRODUCTION}

When pictures were first transmitted via submarine cable between London and New York, one of the first uses of visual imagery were in the newspaper industry. The early 1920s saw the introduction of the Bartlane cable picture transmission machine [1], which cut the time, it took to send a picture across the Atlantic from more than a week to less than three hours. Pictures were coded for cable delivery and then reconstructed at the receiving end using specialized printing equipment. This was how it was sent and how it was printed on a telegraph printer with halftone typefaces [2]. This research yielded a collection of 35 global scope maps showing high-resolution repeat coverage of Mars up to Martian Year 31 under various temporal and viewing situation constraints. These can be used in supplemental content as well as on the internet [3]. The collection of printing procedures and the distribution of pressure levels were two of the first issues in enhancing the visual consistency of these early digital pictures [4].
The printing process used to receive was phased out at the end of 1921 in favor of a photographic replication procedure centered on tapes perforated at the telegraph receiving terminal. In the early 1960s, the first computers capable of performing practical image processing tasks emerged. The availability of such computers, as well as the start of the space programme, coincided with the birth of what we now term digital image processing [5]. It required the convergence of those two technologies to put the promise of digital image processing concepts into sharper view.

The Jet Propulsion Laboratory [6] began working on using computing technology to improve photographs from space probes in 1964, when images of the moon sent by Ranger 7 were processed by a device to fix different forms of image distortion found in the on-board television camera. This is perhaps the first time a US satellite has captured a picture of the moon. The imaging lessons gained with Ranger 7 were used to refine

*Corresponding Author:sureshk@eee@gmail.com / g.s.nila@gmail.com

Received: 20.01.2022 Accepted: 20.02.2022

Published on: 22.02.2022

Suresh Kumar et al., 
and reconstruct photographs from the Surveyor lunar projects, the Mariner series of flyby missions to Mars, the Apollo manned flights to the moon, and other missions Figure 1.

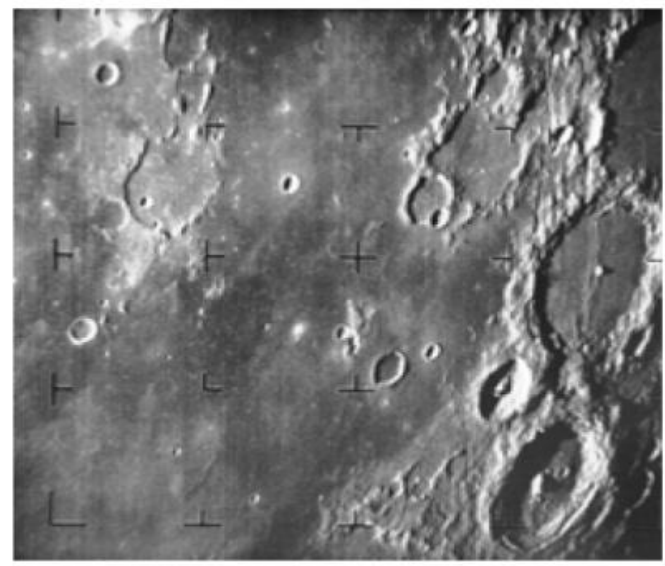

Figure 1 First Picture of the Moon by a U.S. spacecraft [4]

Remote sensing, which usually involves many bands in the visible and infrared areas of the continuum, is another important field of visual processing. NASA's LANDSAT satellite has socalled thematic bands. LANDSAT's main objective is to collect and relay photographs of the Earth from space in order to observe the planet's environmental conditions. The bands are measured in wavelengths, with one metre equaling 10-6 metres. Take note of the features and applications of each band. When the sun was around $10^{\circ}$ above the northwestern horizon, the initial photograph of the mask (frame number 35A72) was shot. As a consequence, the right side is shadowed and no clarity can be seen. As DiPietro and Molenaar discovered the second picture (70A13), which had the sun 17 degrees higher in the atmosphere, they noticed that the face had a strong degree of bilateral symmetry, with what seemed to be a second eye socket and the mouth extension. A pair of crossed lines above the eyes, fine structure in the mouth that others have pointed to as teeth, and long lateral stripes around the face while contrasting carefully reconstructed and improved photographs [7] of the face from $35 A 72$ and 70A13. Several such objects have been recognized for their geometrical regularity and fine detail. I was taken aback by the image processing performance. If these were merely degraded landforms, certain characteristics would not be expected to appear when one studied them more closely. Granted, the features were close to the sensor's resolution maximum of around 50 meters per pixel. They were, however, visible in both 35A72 and 70A13. Random noise, sensor errors, or image processing artifacts did not seem to be the source of these structures (Figure.2).
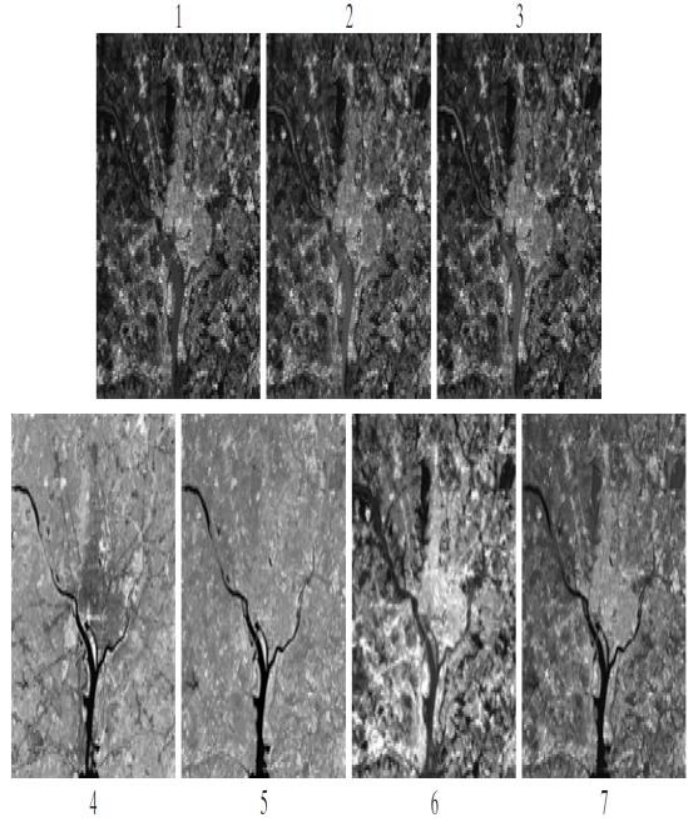

Figure 2 LANDSAT satellite images of the
Washington, D.C. area [4]

\section{Imaging in the Microwave Band}

Radar is the most popular application of imaging in the microwave band. The potential of imaging radar to gather data over nearly any area at any moment, independent of weather or ambient lighting circumstances, is its distinguishing characteristic. Any radar waves can see through clouds and, under certain circumstances, trees, ice, and very dry desert. In certain instances, radar is the best way to explore areas of the Earth's atmosphere that are unavailable. An imaging radar functions similarly to a flash camera in that it generates its own light (microwave pulses) to illuminate a certain region on the ground and capture a snapshot picture. Radar records the photographs using an antenna and optical computer processing [9] rather than a camera lens. Only the microwave energy that was transmitted back into the radar antenna can be seen in a radar signal. A spaceborne radar picture of southeast Tibet's is rough mountainous region about 90 kilometers east of Lhasa. A big valley of the Lhasa River in the lower right corner is inhabited by Tibetan farmers and yak herders, and includes the village of Menba. The valley floors are around $4300 \mathrm{~m}(14,000 \mathrm{ft})$ above sea level, while the mountains in this region hit around $5800 \mathrm{~m}$ $(19,000 \mathrm{ft})$ above sea level. Take note of the image's transparency and detail, which are unaffected by clouds or other atmospheric factors that would usually obstruct photos in the visual band Figure 3. 


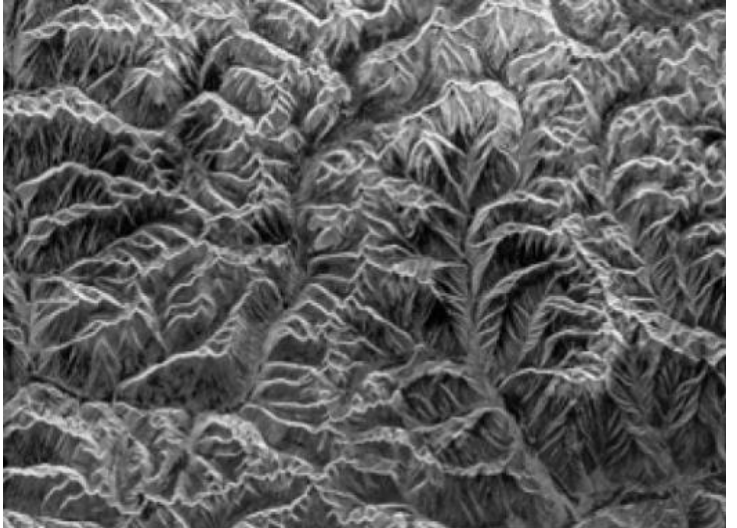

Figure 3 Spaceborne radar images of mountain in southeast Tibet [4]

\section{Imaging in the Radio Band}

The main uses of imaging in the radio band, as with imaging at the other end of the continuum (gamma rays), are medicine and astronomy. Magnetic resonance imaging (MRI) is a medical[10] procedure that uses radio waves (MRI). A patient is placed in a strong magnet and radio waves are sent across his or her body in brief bursts [11]. Each pulse triggers the patient's tissues to release a response pulse of radio waves. A machine determines the source of these signals as well as their frequency, resulting in a twodimensional image of a part of the patient. MRI images can be generated in any aircraft. The last picture on the right is a radio band image of the Crab Pulsar. Photos of the same area captured in several of the bands mentioned earlier are also seen for an important contrast. It's worth noting that each picture depicts the Pulsar in a very different light[3].

\section{Other Imaging Modalities are Used}

While electromagnetic imaging is by far the most common, there are a range of other imaging modalities that are equally significant [12]. Acoustic imaging, electron microscopy, and synthetic (computer-generated) imaging are all included in this portion. Sound-based imaging is used in mineral research, industry, and medicine. Echo at the low end of the sound range (hundreds of Hertz) is used in geological applications, whereas ultrasound is used in some fields for photography (millions of Hertz). Mineral and oil discovery are the most significant industrial uses of image processing in geology. One of the most popular methods for acquiring images over land is to use a big truck with a large flat steel plate. The truck presses the plate against the deck, and the truck vibrates at a frequency of up to $100 \mathrm{~Hz}$. The density of the atmosphere under the soil determines the frequency and intensity of the returning sound waves. These are then processed by a robot, and photographs are created as a result. The energy source for marine acquisition is normally two air guns pulled behind a bow. Hydrophones are mounted in cables that are either towed behind the ship, laid on the ocean floor, or hang from buoys to track returning sound waves (vertical cables). The two air guns are alternately pressurized to a pressure of $2000 \mathrm{psi}$ before being shot [13]. The ship's relentless motion creates a transversal pattern of motion, which is combined with the returned sound waves to create a 3-D map of the Earth's composition below the ocean's surface. The efficiency of seismic imaging algorithms is measured against a cross-sectional representation of a well-known 3D model. A hydrocarbon (oil and/or gas) trap is shown by the arrow. Since the density difference in the target area is higher, it is lighter than the neighboring layers [14].

\section{Image Acquisition Using a Single Sensor}

A single sensor element consists of photodiode, which is made of silicon materials and has an output voltage waveform proportional to light, is perhaps the most well-known sensor of this kind. Selectivity is improved by using a filter in front of a sensor. A green (pass) filter placed in front of a light sensor, for example, favours light in the green continuum. As a result, the sensor sensitivity for green light would be higher than for other visible spectrum elements. There must be relative displacements in both the $\mathrm{x}$ - and $\mathrm{y}$-directions between the sensor and the field to be imaged in order to produce a 2-D picture using a single sensor. A high-precision scanning setup in which a film negative is placed on a drum that rotates mechanically to provide displacement in one direction. The single sensor is attached to a lead screw that allows for perpendicular motion. Since mechanical motion can be precisely regulated, this approach is a low-cost (but time-consuming) way to acquire high-resolution pictures. A flat bed is used in other related mechanical arrangements, with the sensor rotating in two linear directions. Micro densitometers are a term used to describe mechanical digitizers of this kind. A laser source is aligned with the camera in another case of imaging with a single sensor. Moving mirrors are used to guide the transmitted laser light onto the sensor and monitor the outgoing beam in a scanning pattern. This setup can also be used to capture photos with strip and array sensors, which are covered in the next two pages[15].

\section{Global Mars coverage}

The various references are initially overlooked, and a histogram of the repeat picture coverage of 
the martian surface is computed instead. The resolution thresholds are $20 \mathrm{~m}$ and $100 \mathrm{~m} /$ pixel, respectively. Six images with a resolution finer than $100 \mathrm{~m}$ and four images with a resolution finer than $20 \mathrm{~m} /$ pixel occupy the martian terrain on average. Furthermore, less than a third of the martian surface has been mapped with a resolution finer than $20 \mathrm{~m} /$ pixel, a figure that drops to 7.43 percent when photographs with a resolution between 20 and $100 \mathrm{~m} /$ pixel are included. These figures show that it continues to be commonly possible to complete a comprehensive same-area task. Maps depicting the total coverage of resolutions of less than $20 \mathrm{~m}$, between 20 and $100 \mathrm{~m}$, and less than $100 \mathrm{~m}$. Many of the digitizing phenomena that are expected to be observed in reality are based on the sampling concepts presented. When you have a clear understanding of frequency information, you will build on these concepts. The frequency domain is discussed in great depth. In the sense of image acquisition, the principles of sampling and aliasing effects are also relevant. The ideas presented serve as the foundation for pixel neighborhoodbased processing techniques. Most image enhancement and reconstruction techniques use neighbourhood processing processes Figure 4.

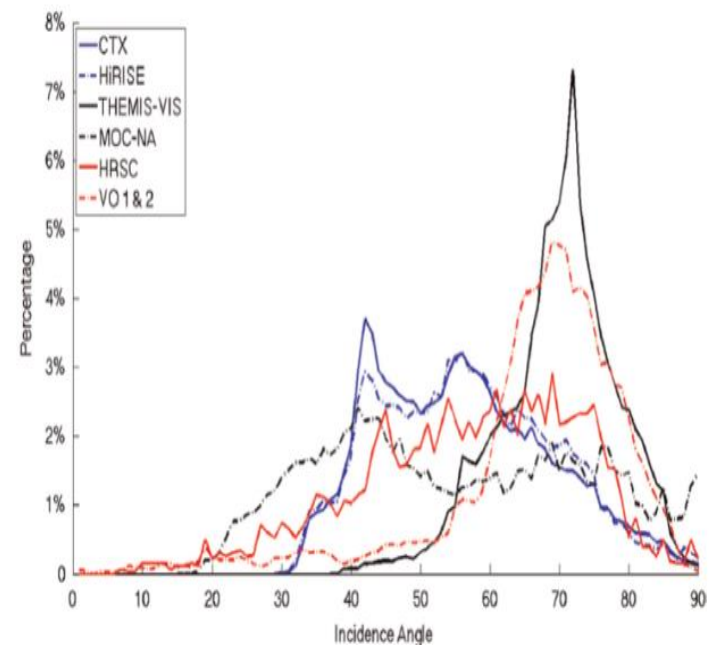

Figure 4: Image Coverage in various points [6]

\section{Single-instrument coverage}

It's possible to see how much attention each camera has received. The orbiter cameras may be divided into two groups, with the first targeting to image small martian surface regions with very high resolution (MOC-NA, HIRISE), and the second aiming to image the whole surface of Mars at the cost of resolution (MOC-NA, HIRISE) (THEMIS-VIS, HRSC, CTX). HRSC has the most detailed mapping of the Martian surface with a resolution of more than $100 \mathrm{~m} /$ pixel, while CTX has the most detailed mapping with a resolution of more than 20 $\mathrm{m} /$ pixel[6]. Furthermore, THEMIS-VIS has an 82.01 percent coverage due to its long life, including an additional source of global Mars photography. Overall, the figures show that for a significant portion of Mars, at least three separate imaging sources with resolutions finer than 100 $\mathrm{m} /$ pixel can be detected. MOC-NA and HiRISE offer additional outlets for areas of expanded science concern, while Viking Orbiter allows for the study of the temporal development of around $23 \%$ of the martian surface during the last 20 Martian Years. Figure 5 shows the surface coverage obtained by each high-resolution orbiter camera[7].

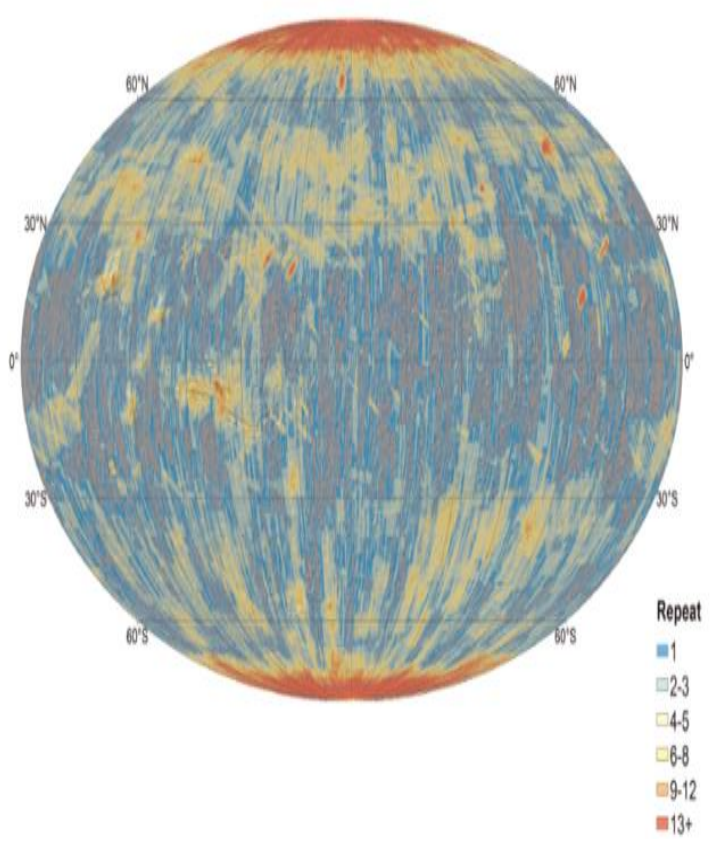

Figure 5The overall repeat coverage of Mars with resolution $20-100 \mathrm{~m} / \mathrm{pixel}$ [7]

\section{CONCLUSION}

The year and season they were discovered on Mars, as well as the lighting conditions under which they were discovered. The accessible evidence, on the other hand, has two main flaws: sparse stereo coverage and a limited number of local periods under which most photographs were collected. The first issue is anticipated to be marginally alleviated in the immediate future, thanks to the CaSSIS stereo camera onboard the ESA's Trace Gas Orbiter, which will launch in 2016. Its nominal target is to achieve alongtrack stereo coverage of 1.7 percent of Mars with a resolution of 5-10 m/pixel for one Martian year. The second would necessitate loosening the restrictions for sun-synchronous orbiters in potential imaging flights, as well as improving the usable illumination modeling, which would allow for the separation of real surface shifts from luminance differences attributable to various

\section{Suresh Kumar et al.,}


incidence angles. The majority of the findings are presented in the form of a sequence of global coverage charts. These maps would be placed into a Web GIS and made accessible on i-Mars. EU project website at the time released. Our main aim, however, is to use this material, as well as the geological background on Mars, to evaluate the ability of multi-instrument surface change detection for each geographic area on the planet. Finally, the Moon would be subjected to a similar investigation in the future. Understanding the origins of the numerous pictures we include in this book requires a study of illumination and the electromagnetic spectrum. Similarly, the picture model provided the foundation for the homomorphic filtering image enhancement method, as well as the impact of lighting on the form of image histograms. Neighborhood processing is preferred in industrial image processing applications where possible due to its operating pace and ease of deployment in hardware and/or firmware. Finally, the definition of a linear operator, as well as its theoretical and logical strength, would be widely utilized.

\section{REFERENCES}

[1] Yingst R. A., Zimbel V. 2020., Dust cover on Curiosity's Mars Hand Lens Imager (MAHLI) calibration target: Implications for deposition and removal mechanisms,351, 113-122

[2] Michael G. G.,Marques M. 2016. Systematic processing of Mars Express HRSC panchromatic and colour image mosaics: Image equalisation using an external brightness reference,Planet. Space Sci., 121, pp. 18-26

[3] Foroutan M., Zimbelman J. R. 2020, Evaluation of large data sets for Transverse Aeolian Ridges (TARs) on Earth and Mars,Planet. Space Sci., 189, pp. 104-126

[4] Pina, P., Marques, J. S., Benavente N. 2012, Mapping Mars in detail : Contributions from pattern recognition and image analysis, $213,29-31$
[5] Malaya Kumar Biswal M., Vishnu, S., Devika S Kumar., Sairam M. 2019, Human mars mission architecture plan to settle the red planet with 1000 people, arXiv, pp.11231146

[6] Mahaffy P. R., Benavente 2012., The sample analysis at mars investigation and instrument suite, Space Sci. Rev., 170, 401478

[7] Verseux, C., Baqué, M., Lehto K., De Vera, J. P. P., Rothschild, L. J., Billi D. 2016, Sustainable life support on Mars - The potential roles of cyanobacteria, Int. J. Astrobiol., 15, pp. 1065-1092

[8] Suresh Kumar, P., Meenakshi, S., Venkatesh A.2020, Performance Analysis of High Voltage Intelligent Supervisory Systems Using Neural Networks, Int. J. Adv. Sci. Eng., 7(2)1525-1532

[9] Suresh Kumar, P., Meenakshi, S., Prathap G. 2019. Some Investigations on Heterogeneous Deep Learning Network using Stable Election Protocol with Region Based Energy Conservation, Int. J. Adv. Sci. Eng., 6(2)1361-1369

[10] Meenakshi, S. Suresh Kumar, P., Ramsanjay S.2020. Soft Computing Techniques based Digital Adoptive Controllers with Intelligent System for Switched Reluctance Motor, Int. J. Adv. Sci. Eng. 6(3) 1430-1438

[11] Suresh Kumar, P., Meenakshi S.2020, Performance Analysis of FPGA based Implementation of FFT Architecture with Pruning Algorithm for Industrial Applications, Int. J. Adv. Sci. Eng., 7(2)17701775,

[12] Suresh Kumar, P., Meenakshi, S., Prathap, G., Nirmala G.2020, Soft Computing Techniques Based MRAC with Intelligent Control for Stability Analysis, Int. J. Adv. Sci. Eng., 6(3)1430-1438 